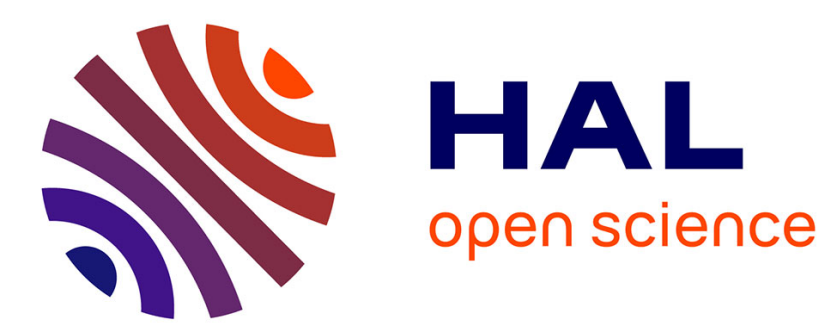

\title{
Design of Optimal Short OFDM-Type Windows
}

Pierre Siohan, Didier Pinchon

\section{To cite this version:}

Pierre Siohan, Didier Pinchon. Design of Optimal Short OFDM-Type Windows. 2019. hal-02111698

\section{HAL Id: hal-02111698 \\ https://hal.science/hal-02111698}

Preprint submitted on 26 Apr 2019

HAL is a multi-disciplinary open access archive for the deposit and dissemination of scientific research documents, whether they are published or not. The documents may come from teaching and research institutions in France or abroad, or from public or private research centers.
L'archive ouverte pluridisciplinaire HAL, est destinée au dépôt et à la diffusion de documents scientifiques de niveau recherche, publiés ou non, émanant des établissements d'enseignement et de recherche français ou étrangers, des laboratoires publics ou privés. 


\title{
Design of Optimal Short OFDM-Type Windows
}

\author{
Pierre Siohan ${ }^{1}$ and Didier Pinchon ${ }^{2}$ \\ ${ }^{1}$ Independent Researcher, Brittany, France \\ ${ }^{2}$ Institut de Mathématiques de Toulouse, Université Paul Sabatier , \\ Toulouse, France
}

February 20, 2019

\begin{abstract}
One key issue of the fifth mobile communication generation $(5 \mathrm{G})$ is to elaborate an appropriate new waveform. Ideally, this waveform should preserve the two main advantages of the Orthogonal Frequency Division Multiplexing (OFDM) scheme, namely low latency and complexity. Along that line of thought, we propose in this paper an OFDM-type window, i.e. identical symbol time duration and equivalent spectral efficiency as OFDM. This optimal waveform with respect to the time-frequency localization criterion is analyzed using a Filtered MultiTone (FMT) framework. Our design examples illustrate its huge benefits compared to OFDM and we also compare its behavior with respect to the Root Raised Cosine window.
\end{abstract}

Keyword: FMT,OFDM,Perfect Reconstruction,Root Raised Cosine, Time-Frequency Localization

\section{Introduction}

The future 5G cellular communication system with its ambitious requirements [1, 2] has led to a wide variety of requirements concerning the physical modulation schemes see e.g. [3]. Indeed, it is argued that the 4G Cyclic-Prefix Orthogonal Frequency Division Multiplexing (CP-OFDM) is not suitable for the diversity of services envisioned for 5G. Actually, CPOFDM limits are due to its bad frequency behavior that strongly impact its performance in asynchronous scenarios as well as in high mobility contexts.

On the other hand, CP-OFDM big advantage is to use for each transmitted multi-carrier (MC) symbol a simple rectangular window ensuring by the way low computational complexity and low latency. As a matter of consequence, a slight modification of the CP-OFDM, known as the Weighted Overlap and Add (WOLA)-OFDM [4], [5], has gained a large interest into the $3 \mathrm{GPPP}$ standardization community. In this latter scheme the plain rectangular OFDM window is modified introducing soft edges at both sides using the Root Raised Cosine (RRC) function. In this paper our aim is two-fold: 
1. To highlight the fact that the discrete version of the RRC function, here named dual RRC (dRRC), also provides a perfect-reconstruction (PR) solution for Filtered MultiTone (FMT) MC transmission systems.

2. To analyze the dRRC prototype filter in terms of its time-Frequency Localization (TFL) properties and to compare it with optimal TFL short windows, i.e. also limited to one MC symbol duration, leading to Optimal TFL (OTFL)-OFDM systems.

Doing so, we significantly extend some of our previous works. Indeed, in [6, 7], we have derived explicit expressions leading to the maximization of the TFL criterion for PR FMT prototype filters of length $L=N$, with $N$ the expansion/decimation factor of the corresponding $M$ - subcarrier FMT system with $M<N$. However, these optimal (or nearly optimal) solutions, reused afterwards to derive Weighted Cyclic Prefix (WCP)-OFDM [8], Pulse Shaped OFDM (P-OFDM) [9] and also, using a duality property, Faster Than Nyquist (FTN) MC systems [10], are only valid for a subset of $M$ and $N$ values since the parameters settings in $[6,7]$ only consider the case of FMT systems having a maximum spectral efficiency (SE). More precisely, in $[6,7]$, the investigation is limited to the case where, setting $\Delta=$ $\operatorname{gcd}(N, M)$, it is imposed that $N_{0}=M_{0}+1$ with $M=\Delta M_{0}, N=\Delta N_{0}$. As noted in [9], this subset of solutions does not cover important applications as for instance the ones related, for frame compatibility reasons, to $5 \mathrm{G}$ waveforms constraints.

In this paper, we include the case where $M$ and $N$ can take any value such that $M<$ $N \leq 2 M-1$. The PR solutions for the corresponding FMT filterbank can, as in [6, 7], be expressed using an angular representation. As we only here consider short $L=N$ windows, the optimization problem only involves $N-M$ angular parameters. Nonetheless, this nonlinear optimization problem may become quickly untractable for high values of $N-M$. For instance, referring to the design of $5 \mathrm{G}$ waveforms for the $20 \mathrm{MHz}$ band we have $M=2048$ and $N=2192$, meaning that 144 parameters have to be optimized.

To extend the initial results of $[6,7]$, we, again, take advantage of the nice features of our compact representation method [11]. That means the angular parameters are expressed using a $d$-coefficients polynomial representation. Then, we show that optimal (or nearly optimal) results can be obtained with $d=1$, or, at most $d=2$.

On another hand, as the dRRC window also provides good TFL results, in order to fully validate its interest, we propose an in depth comparative analysis between both windows.

Our paper is organized as follows. In Section 2, we recall the background concerning the RRC function and FMT systems and show that the discrete-time RRC sequence leads to a PR FMT system. In Section 3, we present the optimization method of the OTFL-OFDM window. Comparisons between the dRRC and OTFL windows are provided in Section 4.

\section{Background}

\subsection{RRC and dual RRC functions}

Since it has been used to illustrate Nyquist's first criterion for interference-free transmission, the RRC function holds a key position in the communication field. Originally expressed in 
continuous-time, the RRC function can be formulated using the following Fourier transform pair

$$
\begin{array}{r}
r(u, U)=\sqrt{U} \frac{4 \alpha U u \cos (\pi(1+\alpha) U u)+\sin (\pi(1-\alpha) U u)}{\left(1-(4 \alpha U u)^{2}\right) \pi U u} \\
R(v, V)= \begin{cases}\frac{1}{\sqrt{V}}, & |v| \leq(1-\alpha) \frac{V}{2}, \\
\frac{1}{\sqrt{V}} \cos \left(\frac{\pi}{2 \alpha}\left(\frac{|v|)}{V}-\frac{1-\alpha}{2}\right)\right), & (1-\alpha) \frac{V}{2}<|v| \leq(1+\alpha) \frac{V}{2}, \\
0, & (1+\alpha) \frac{V}{2}<|v|\end{cases}
\end{array}
$$

Both functions can be used either in time and frequency domain as illustrated in Table 1.

\section{$2.2 \quad$ CP-OFDM and FMT modulations}

Initially introduced for single carrier transmission, the RRC case was popularized afterwards for the FMT case [12], i.e. a MC system where the transmitted signal $s(t)$ is modulated using a Gabor family

$$
s(t)=\sum_{m, n} c_{m, n} p_{m, n}(t) \text { with } p_{m, n}(t)=p(t-n T) e^{j 2 \pi m F t}
$$

with $p(t)$ the prototype function and $c_{m, n}$ the complex symbols to be transmitted. Let for OFDM or, equivalently, for the Discrete MultiTone (DMT) system, $T=T_{0}$ denote the MC symbol duration and $F=F_{0}=1 / T_{0}$ the frequency spacing between subcarriers. Then $p(t)$ is simply the rectangular window function, i.e. $p(t)=\Pi_{T_{0}}(t)$.

In [12], the goal being to reduce the high DMT overlapping between subcarriers, the proposal is to increase the frequency subspacing multiplying it by $(1+\alpha)$ and to choose a RRC prototype function in accordance $p(t)=r\left(t,(1+\alpha) F_{0}\right)$, i.e. with a better frequency behavior than the one obtained by the OFDM rectangular window.

The discrete-time counterpart of this filterbank modulation system reads as follows

$$
s[k]=\sum_{m, n} c_{m, n} p_{m, n}[k] \text { with } p_{m, n}[k]=p[k-n N] e^{j 2 \pi \frac{m}{M}}
$$

with $M$ the subcarrier number and $N$, with $N>M$, the expansion/decimation factor of the FMT filterbank implementation.

Denoting by $S_{e}$ the SE, OFDM reaches with $S_{e}=1$ the maximum value for a MC orthogonal system, while for FMT, as the subcarrier spacing is increased by a factor $N / M>$ 1 , we have $S_{e}=\frac{M}{N}<1$. Then, to reduce this loss in spectral efficiency, $N$ has to be chosen as close as possible from $M$. This means that the roll-off factor of the corresponding RRC prototype filter $\alpha$ must tends to zero, thus requiring a longer filter, which means increase complexity and latency for the RRC FMT system. Concerning OFDM, in practice, symbols are either Zero Padded (ZP), leading to a $N$-length ZP-OFDM symbol with prototype filter $p_{z p}[n]=\Pi_{M}[n]$ for $0 \leq n \leq M-1$ and 0 in the $(N-M)$-length guard interval, or they are cyclically extended with a Cyclic Prefix (CP) leading to CP-OFDM and a prototype filter $p_{c p}[n]=\Pi_{N}(n]$. 


\begin{tabular}{|c|c|c|}
\hline & $\mathrm{RRC}(\alpha)$ & $\mathrm{dRRC}(\alpha)$ \\
\hline$r(t)$ & $r\left(t, F_{0}\right)$ & $R\left(t, T_{0}\right)$ \\
\hline$R(\nu)$ & $R\left(\nu, F_{0}\right)$ & $r\left(\nu, T_{0}\right)$ \\
\hline
\end{tabular}

Table 1: RRC and dRRC functions for a transmission at rate $T_{0}$ or $T=(1+\alpha) T_{0}$.

So differently from FMT, the redundancy is introduced in the time domain.

However if, in (3), we set $T=(1+\alpha) T_{0}=\frac{N}{M} T_{0}$ and $F=F_{0}$ we get a MC system, we also call FMT for simplicity ${ }^{1}$, the spectral efficiency of which is again $S_{e}=\frac{M}{N}$ and also equal to the one of ZP or CP-OFDM systems with guard interval, or CP length, equal to $N-M$. One can get a simple discrete-time causal prototype filter for this FMT system. Let us start setting $r(t)=R\left(t, T_{0}\right)$. Shifting to the right to make this expression causal and normalizing, we get $r_{c}(t)=\sqrt{T_{0}} r\left(t-(1+\alpha) \frac{T_{0}}{2}\right)$. Then, denoting by $T_{s}$ the sampling period, we get with $r_{c}[n]=r\left((n+1) T_{s}\right)$, the causal dRRC prototype filter given by ${ }^{2}$

$$
r_{c}[n]= \begin{cases}\sin \left(\frac{(n+1) \pi}{2(N-M+1)}\right), & 0 \leq n \leq N-M-1, \\ 1, & N-M \leq n \leq M-1, \\ \sin \left(\frac{(N-n) \pi}{2(N-M+1)}\right), & M \leq n \leq N-1 .\end{cases}
$$

Notice that, in order to avoid the two extreme impulse response coefficients for $n=0$ and $N-1$ be equal to 0 , instead we have here $r_{c}[-1]=0$ and a roll-off interval in discrete-time such that $\alpha=(N-M+1) / M$.

\subsection{PR conditions for FMT systems}

For general values of $M$ and $N$ with $M<N$, the PR relation for a prototype filter $P(z)=$ $\sum_{n=0}^{L-1} p[n] z^{-n}$ of length $L$ may be written (cf. [6, equation (2)])

$$
\sum_{\nu} p[k+\nu M] p[k+\nu M+s N]=\delta_{s}, 0 \leq k \leq M-1, s \geq 0
$$

where $\delta_{s}$ is the Kronecker symbol equal to 1 if $s=0$ and equal to 0 elsewhere. In (6), by convention, $p[n]=0$ if $n<0$ or $n \geq L$.

When $L=N$ and for $s>0, p[k+\nu M]=0$ or $p[k+\nu M+s N]=0$ for every value of $k$ and $\nu$. For $s=0,(6)$ is equivalent to

$$
\begin{aligned}
& p[k]^{2}+p[k+M]^{2}=1,0 \leq k \leq N-M-1, \\
& p[k]^{2}=1, N-M \leq k \leq M-1
\end{aligned}
$$

Note at this step that,

\footnotetext{
${ }^{1}$ Strictly speaking, as FMT corresponds to a system with enlarged subcarrier spacing w.r.t. OFDM and CP-OFDM, dual FMT (dFMT) could be a more appropriate denomination.

${ }^{2}$ Though equation (5) is presented into a background section, we have not found a previous example of this discrete time RRC formulation.
} 
- The ZP-OFDM transmitter window, $p_{z p}$, satisfies the PR condition (6) for an FMT system with an identical $p_{z p}$ receiver window,

- The CP-OFDM system does not satisfy the PR condition but biorthogonality conditions with a $M$-length prototype filter $q[n]=\Pi_{M}[n]$ at the receiver side [8],

- The dRRC prototype filter (cf. (5)) satisfies the PR conditions.

However, ZP or CP-OFDM prototype filters are not satisfactory with regard to their bad frequency behavior, and our goal in here is to find PR prototype filters being optimal with respect to the TFL criterion and to analyze the dRRC filter in this respect.

\subsection{Time-frequency localization criterion}

Following Doroslovački [13], the time-frequency localization $\xi$ of a length $L$ filter $P(z)=$ $\sum_{n=0}^{L-1} p[n] z^{-n}$ is defined by the following formulas:

$$
\begin{aligned}
T & =\frac{\sum_{n}\left(n-\frac{1}{2}\right)(p[n]+p[n-1])^{2}}{\sum_{n}(p[n]+p[n-1])^{2}}, \\
\|P\| & =\left(\sum_{n} p[n]^{2}\right)^{\frac{1}{2}}, \\
m_{2} & =\frac{1}{4\|P\|^{2}} \sum_{n}\left(n-\frac{1}{2}-T\right)^{2}(p[n]+p[n-1])^{2}, \\
M_{2} & =\frac{1}{\|P\|^{2}} \sum_{n}(p[n]-p[n-1])^{2}, \\
\xi & =\frac{1}{\sqrt{4 m_{2} M_{2}}} .
\end{aligned}
$$

with $m_{2}$ and $M_{2}$ the second order moments in time and frequency, respectively. For this discrete time measure, as also the case for the counterpart measure used for continuous time signals, we have $0 \leq \xi \leq 1$.

For a $K$-length rectangular window $(\mathrm{RW}), \Pi_{K}$, it can be checked that

$$
\xi_{R W}[K]=\frac{\sqrt{3 K}}{\sqrt{2 K^{2}-3 K+4}} .
$$

From which we derive the TFL measures for ZP-OFDM, $\xi_{z p}[M]=\xi_{R W}[M]$, and CP-OFDM, $\xi_{c p}[M]=\xi_{R W}[N]$.

Expressions which illustrate the fact that, in both cases, the TFL tends to zero when $M$ goes to infinity. 


\section{TFL optimization using the compact representation}

\subsection{Symmetric compact representation}

In order to optimize the TFL criterion, we choose $p[k]=1$ for $N-M \leq k \leq M-1$, and for $0 \leq k \leq N-M-1,(7)$ implies that there exists an angle $\theta_{k}$ such that $p[k]=\cos \theta_{k}$ and $p[k+M]=\sin \theta_{k}$. The optimization program may then consider the $N-M$ variables $\theta_{k}, 0 \leq k \leq N-M-1$ as optimization variables.

Several trials have shown that the optimized $\theta_{k}$ values have a regular behavior with respect to $k$, and therefore a compact representation method as defined in [11] may be used. We thus consider a function of $u(x), x \in[0,1]$ depending on $d$ parameters and we set $\theta_{k}=\frac{\pi}{2} u\left(\frac{k+1}{N-M+1}\right), 0 \leq k \leq N-M-1$. $d$ is called the degree of the compact representation. In this way the $N-M$ variables for optimization of the TFL criterion are replaced by the $d$ parameters of the function $u(x)$, which greatly simplifies the computation task when $d \ll N-M$.

After several trials, we observe that the optimal filter $P(z)=\sum_{k=0}^{N-1} p[k] z^{-k}$, for any given values of $M$ and $N$, has the linear phase property, that is $p[k]=p[N-1-k], 0 \leq k \leq$ $N-1$. This implies that the function $u(x)$ of a compact representation could be restricted to functions such that $\cos \theta_{k}=\sin \theta_{N-1-k}$ and because $\theta_{k}=\frac{\pi}{2} u\left(\frac{k+1}{N-M+1}\right), u(1-x)=1-u(x)$ for values $x=\frac{k+1}{N-M+1}, 0 \leq k \leq N-M-1$.

So it is justified to choose $u(x)$ such that $u(1-x)=1-u(x)$ for every $x$ in $[0,1]$. The chosen compact representation, called the symmetric compact representation (SCR), may thus be defined by

$$
u(x)=\frac{1}{2}+\sum_{i=0}^{d-1} e_{i}\left(x-\frac{1}{2}\right)^{2 i+1},
$$

where $d$ is the degree of the compact representation and $e_{i}, 0 \leq i \leq d-1$ its $d$ coefficients, used as variables in the optimization process.

As a matter of example, it can be checked that the dRRC filter corresponds to a SCR with $d=1$ with coefficient $e_{0}=1$ while the Meyer auxiliary function [14], [5] $v(x)=$ $x^{4}\left(35-84 x+70 x^{2}-20 x^{3}\right)$ can be seen as a SCR with $d=4$ and coefficients given by

$$
e_{0}=\frac{35}{16}, e_{1}=-\frac{35}{4}, e_{2}=21, e_{3}=-20
$$

\subsection{A particular case: $N=M+1$}

When $N=M+1$, equations (8) reduce to $p[0]^{2}+p[M]^{2}=1$ and so the PR filter with length $M+1$ is equal to

$$
P(z)=\cos a+\sum_{k=1}^{M-1} z^{-k}+\sin a z^{-M}
$$


The TFL criterion $\xi(M, a)$ for this filter is thus a function of $M, \cos a$ and $\sin a$. As the symmetric filter $\sigma P(z)$ defined by

$$
\sigma P(z)=z^{-M} P(1 / z)=\sin a+\sum_{k=1}^{M-1} z^{-k}+\cos a z^{-M},
$$

has the same TFL value, that means it remains invariant when exchanging $\cos a$ and $\sin a$, i.e. by the transformation $a \rightarrow \frac{\pi}{2}-a$. Setting $x=\cos a$ and $y=\sin a, \xi(M, a)$ can be expressed as a function of $x+y=S$ and $x y=\left(S^{2}-1\right) / 2$.

Then, a direct computation, using equations (9) to (13), allows to prove that, for every $M$ value greater or equal 2 , we have

$$
\xi(M, a)=\frac{M \sqrt{3(2 M-2+S)}}{\sqrt{(2-S) F(M, S)}},
$$

where

$$
\begin{aligned}
S= & \cos a+\sin a, \\
F(M, S)= & 3 M^{2} S^{4}+6 M(M-1) S^{3}-6(2 M-1) S^{2}+2(M-2)\left(4 M^{2}-10 M+3\right) S \\
& +2(M-1)\left(2 M^{3}-6 M^{2}+13 M-3\right) .
\end{aligned}
$$

Because

$$
\frac{d \xi(M, a)}{d a}=\frac{d \xi(M, a)}{d S} \frac{d S}{d a}=\frac{d \xi(M, a)}{d S}(\cos a-\sin a),
$$

$\xi(M, a)$ may have a local extremum at $a=\frac{\pi}{4}$, and it is straightforward to check that it is indeed a local maximum. Using the fact that $\xi(M, a)^{2}$ is a rational fraction of $M$ and $S$, it is also possible, but quite cumbersome, to prove that it is a global maximum at $a=\frac{\pi}{4}$ for any given value of $M>2$. Note also that in this case we recover the dRRC prototype filter. Otherwise said, for $N=M+1$, the dRRC is the optimal solution for time-frequency localization. For $M=2$, the best TFL filter is obtained for $a=0$ or $a=\frac{\pi}{2}$, that is for $P(z)=1+z^{-1}$ or $P(z)=z^{-1}+z^{-2}$ for which $\xi(2, a)=1$.

For the value $a=\frac{\pi}{4}$, we check by setting $S=\sqrt{2}$ in (16) that

$$
\xi\left(M, \frac{\pi}{4}\right)=\frac{M \sqrt{3(2+\sqrt{2})}}{\sqrt{2} \sqrt{2 M^{3}-3(2-\sqrt{2}) M^{2}+2(8-3 \sqrt{2}) M-3(2-\sqrt{2})}} .
$$

Similarly to CP-OFDM, when $M$ goes to infinity, the TFL measure tends to zero but not as fast as for CP-OFDM. Indeed, setting $N=M+1$ in (14), it can be checked that, for $\mathrm{ZP}$ and $\mathrm{CP}-\mathrm{OFDM}$ as long as the number of subcarriers is such that $M \geq 8$, the relative improvement brought by the optimal TFL pulse is always above $30 \%$. 


\subsection{Determination of the symmetric compact representation de- gree}

For given $M$ and $N$ with $M+2 \leq N \leq 2 M-1$, let us denote by $\xi_{d}$ the best value of the TFL measure for a SCR of degree $d$.

For $3 \leq M \leq 200$ and $M+2 \leq N \leq 2 M-1$, the values of $\xi_{1}, \xi_{2}$ and $\xi_{3}$ have been computed using the CFSQP program [15] and for this set of parameters $(M, N)$, we have observed that the benefit of an increased degree becomes less and less. Indeed, setting $d>3$ only brings a meaningless gain. Therefore, denoting by $\xi_{\text {opt }}$ the optimal TFL measure, we will assume that, for any $N$-length prototype filter, we have $\xi_{3}=\xi_{\text {opt }}$.

\section{Comparison between the dRRC and OTFL windows}

\subsection{Theoretical dRRC features}

A first property, already recalled before, is that the dRRC prototype filter insures the PR property for FMT modulation systems. We have also seen that for $N=M+1$, the dRRC window is optimal with respect to the discrete-time TFL criterion.

As well-known the weak point for the OFDM window occurs in the frequency domain, otherwise said is related to its second order moment $M_{2}$. In this respect, let us examine what can be expected with $L=N$ windows.

Definition 4.1. For given $M$ and $N$ with $M \geq 2$ and $M<N<2 M$, let us denote the dRRC filter by $P_{\mathrm{dRRC}}(z)=\sum_{n=0}^{N-1} r_{c}[n] z^{-n}$.

We now designate, for the above considered $M$ and $N$ parameters, by $\mathcal{P}_{M, N}$ the set of symmetrical $N$-length PR filters. Then the following theorem holds.

Theorem 4.2. For given $M$ and $N$ such as $M \geq 2$ and $M<N<2 M$

$$
\min \left\{M_{2}(P(z)), P(z) \in \mathcal{P}_{M, N}\right\}=M_{2}\left(P_{d R R C}\right)=\frac{4(N-M+1)}{M} \sin ^{2}\left(\frac{\pi}{4(N-M+1)}\right),
$$

and $P_{d R R C}$ is the only filter belonging to $\mathcal{P}_{M, N}$ reaching this minimum.

Proof is reported in Appendix.

Reaching the optimum $M_{2}$ measure does not obviously mean to be optimum for the TFL criterion as shown in the numerical analysis section.

\subsection{Numerical analysis}

\subsubsection{Performance measure}

Taking now the dRRC prototype filter as our reference, we have computed the relative gain $G_{d}(M, N)$ that can be obtained with the OTFL filters for $d=1$ and $d=3$. In this purpose, the expression

$$
G_{d}(M, N)=\frac{\xi_{d}-\xi_{\mathrm{dRRC}}}{\xi_{\mathrm{dRRC}}}
$$


has been evaluated for $3 \leq M \leq 200$ and $M+2 \leq N \leq 2 M-1$.

We will naturally agree on the fact that the lower these gains are the higher the interest of the dRRC filter is.

Denoting by $\rho=\frac{N-M}{M}$ the relative redundancy of the MC modulation system, we plotted in Figure 1 the graph of the threshold function $f_{3}^{(3)}(\rho)$ where $f_{3}^{\left(M_{0}\right)}(\rho)$ is defined by

$$
f_{3}^{\left(M_{0}\right)}(\rho)=\max \left\{G_{3}(M, N), M \geq M_{0}, \frac{N-M}{M} \leq \rho\right\} .
$$

Of course this function decreases when $M_{0}$ increases.

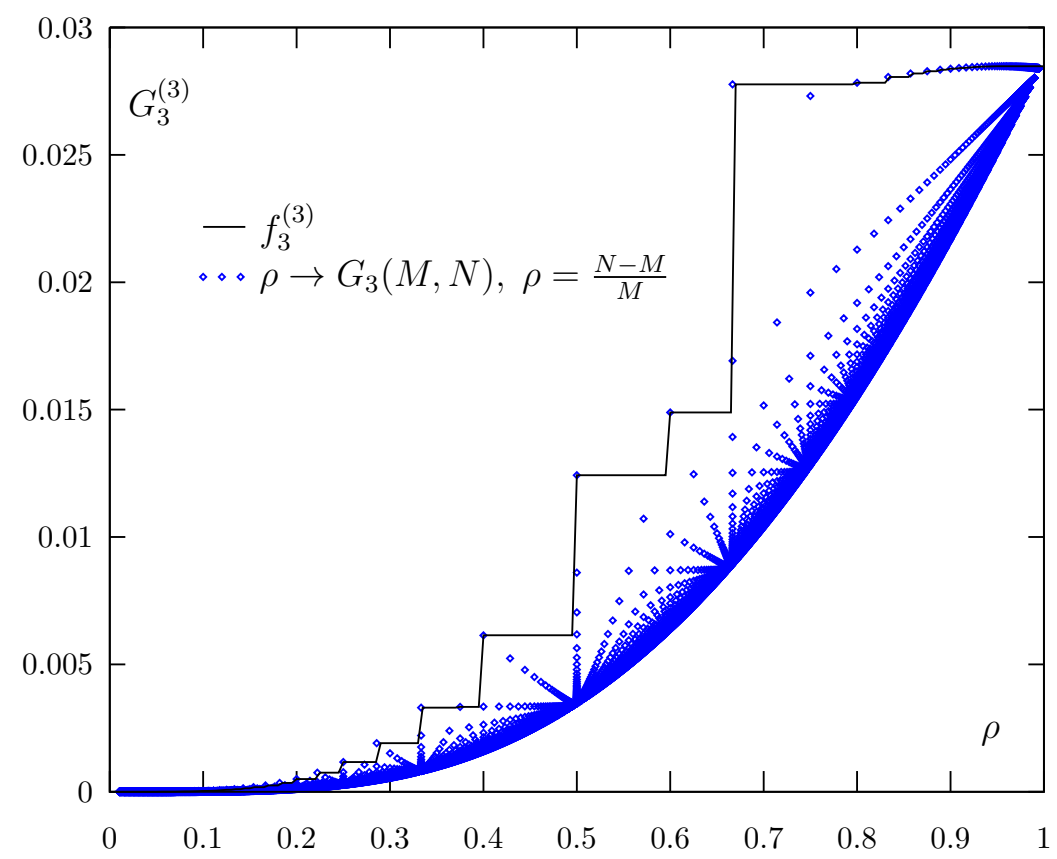

Figure 1: Relative gain $G_{3}(M, N)$ for $3 \leq M \leq 200, M+2 \leq N \leq 2 M-1$.

For threshold gains given in the first column, Table 2 indicates the maximal acceptable value of $\rho$ denoted $\rho_{3}$ for $M_{0}=3$ and $\rho_{20}$ for $M_{0}=20$.

\begin{tabular}{|c|c|c|}
\hline Threshold & $\rho_{3}$ & $\rho_{20}$ \\
\hline $2 \times 10^{-3}$ & 0.33 & 0.39 \\
\hline $5 \times 10^{-3}$ & 0.39 & 0.52 \\
\hline 0.01 & 0.49 & 0.64 \\
\hline
\end{tabular}

Table 2: Some thresholds for $G_{3}(M, N)$.

So the second line in Table 2 indicates that for $M \geq 3$, we obtain $G_{3}(M, N)<5 \times 10^{-3}$ if $\rho<0.39$.

As a matter of example, for $M=128$, we may check that $G_{3}(128, N)<5 \times 10^{-3}$ if $\rho \leq 0.546875$, i.e. $N \leq 198$. More generally, Figure 2 shows the relative gains $G_{1}(128, N)$ and $G_{3}(128, N)$ for $130 \leq N \leq 255$. In particular $G_{1}(128, N) \leq G_{1}(128,255)=1.03 \times 10^{-3}$. 
This proves that for $d=1$, i.e. an identical SCR degree as the dRRC one, there is nearly nothing to expect from an optimal filter as the improvement for $d=1$ is at most of $0.1 \%$. However, we see for $d=3$ that it may be interesting to use the filter obtained by an optimization of the TFL for values of $\rho$ near 1.

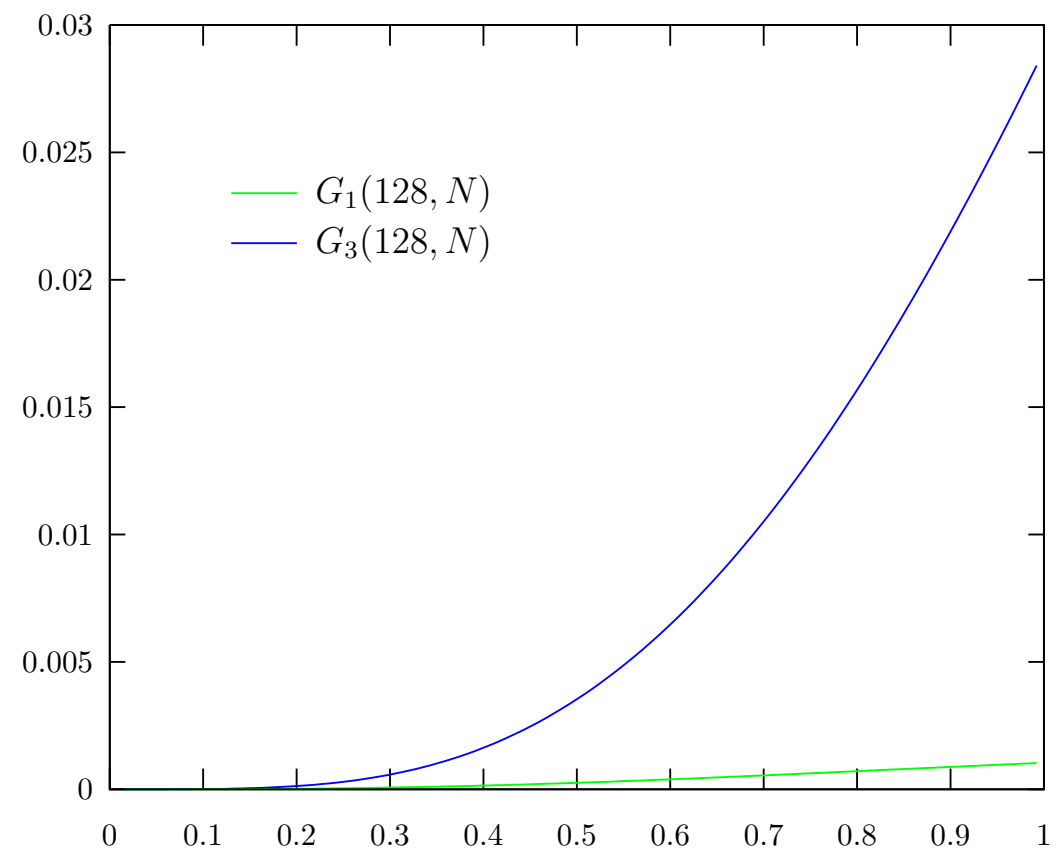

Figure 2: Relative gains $G_{1}(M, N)$ and $G_{3}(M, N)$ for $M=128$ and $130 \leq N \leq 255$.

\subsubsection{Impact of the oversampling ratio}

Increasing the oversampling ratio $\frac{N}{M}$, also means increasing the redundancy $\rho$ and decreasing the spectral efficiency as $\frac{N}{M}=\frac{1}{S_{e}}$. To illustrate the impact of $\rho$, let us compare two examples: $M=128, N=137$ where $\rho=0.0703125$ and $M=128, N=255$ where $\rho=0.9921875$.

In order to get a better balance between second order moments in time and frequency, those are now normalized using a rule proposed in [16] and leading here to

$$
m_{2}^{(N)}=\frac{2}{M^{2}} m_{2}, \quad M_{2}^{(N)}=\frac{M^{2}}{8 \pi^{2}} M_{2} .
$$

In Table 3 it is worth noting that

- In line with theorem 4.2 , for both examples the second order moment in frequency is less for the dRRC filter than for the OTFL one.

- Increasing the oversampling ratio naturally allows us to get better TFL performances but at the price of a higher redundancy.

- The differences in performance between OTFL and dRRC are nearly negligible for the $M=128, N=137$ case. More generally speaking, when considering the different LTE 
settings, i.e. $M=128 \Delta, N=137 \Delta$, with $\Delta=2^{i-1}, 1 \leq i \leq 6$, the TFL measures tend to decrease when increasing $\Delta$ and their relative differences as well. For example, for $M=2048$ and $N=2192$, we get $\xi_{\text {opt }}=0.292985587$ and $\xi_{\text {dRRC }}=0.292985052$, so that $G_{3}(2048,2192)=1.83 \times 10^{-6}$.

\begin{tabular}{|c||c|c|c||c|c|c|}
\hline \multicolumn{1}{|c||}{} & \multicolumn{3}{c||}{$M=128, N=137$} & \multicolumn{3}{c|}{$M=128, N=255$} \\
\hline & dRRC & Opt & Rel. gain & dRRC & Opt & Rel. gain \\
\hline$m_{2}^{(N)}$ & 0.1670191 & 0.1670172 & $-1.97 \times 10^{-5}$ & 0.2613721 & 0.2347256 & $-1.02 \times 10^{-1}$ \\
\hline$M_{2}^{(N)}$ & 0.3991782 & 0.3991804 & $5.38 \times 10^{-6}$ & 0.03124961 & 0.03290138 & $5.28 \times 10^{-2}$ \\
\hline TFL & 0.3081936 & 0.3081944 & $2.69 \times 10^{-6}$ & 0.8805180 & 0.9055301 & $2.84 \times 10^{-2}$ \\
\hline
\end{tabular}

Table 3: Relative gains for $M_{2}^{(N)}, m_{2}^{(N)}$, $\xi$ when $M=128, N=137$ and $M=128, N=255$.

Our results clearly show that there is nearly nothing to expect from OTFL systems w.r.t. dRRC ones when $\rho$ takes low values, as it the case for example for LTE settings. At the contrary, when $\rho$ comes close to 1 , differences are higher and become visible in the time and frequency domains as shown in Figure 3.
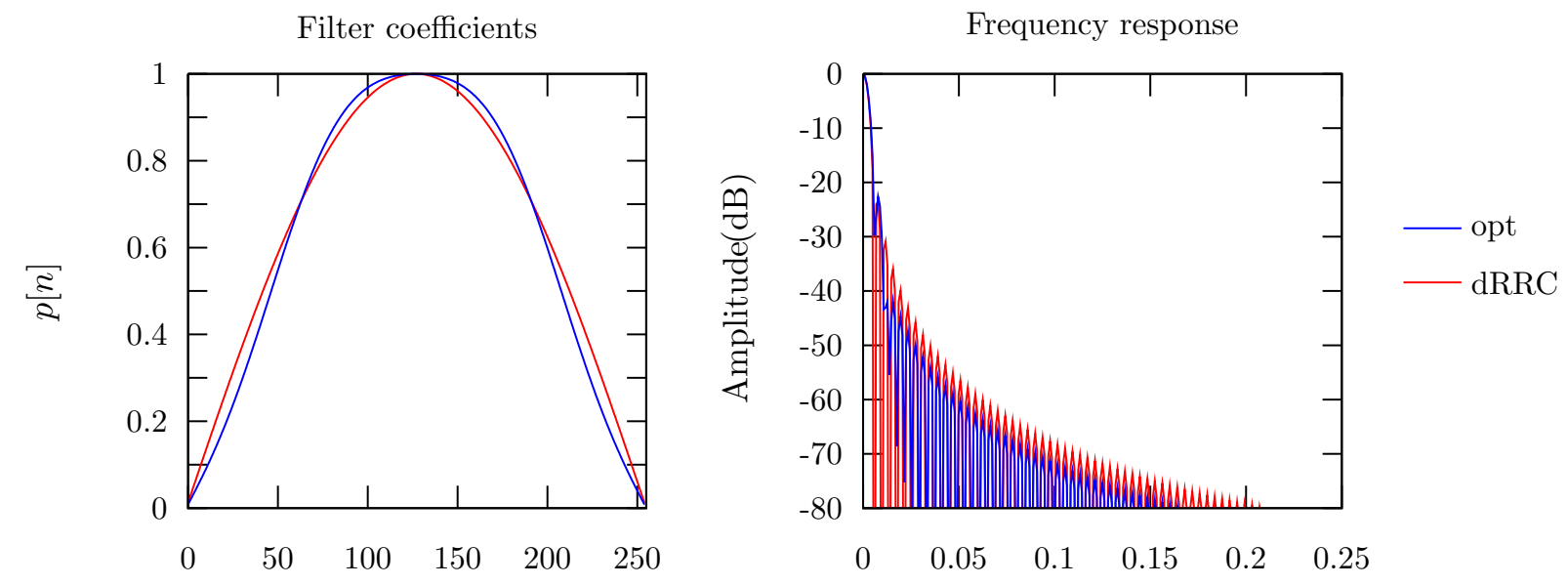

Figure 3: Filter with optimal TFL and dRRC filters for $M=128$ and $N=255$.

Naturally, for FMT or OFDM-type systems, low oversampling ratios are preferred. But, on the other hand, it has been shown recently, cf. e.g. [10], that dual ${ }^{3}$ systems w.r.t. PR FMT systems can be built providing high efficiency MC Faster Than Nyquist systems. For these FTN systems the increase in symbol rate is proportional to $\frac{N}{M}$, making the case $N=2 M-1$ of utmost interest.

\footnotetext{
${ }^{3}$ In practice, keeping the same synthesis and analysis filter banks and exchanging the role of $M$ and $N$ parameters, see [10] for more details.
} 


\subsubsection{Approximation formulas}

Let us now consider values of $M$ and $N$ with $M+1<N<2 M$ with a fixed value of $\rho=\frac{N-M}{M}$ and $M$ tending to infinity : for fixed values of $M_{0}, N_{0}$ with $M_{0}+1<N_{0}<2 M_{0}$, and $\rho=\frac{N_{0}-M_{0}}{M_{0}}$, we choose $M=\Delta M_{0}$ and $N=\Delta N_{0}$ with $\Delta \rightarrow+\infty$. We may observe that $\xi_{\text {opt }}$ is well approximated by

$$
\xi_{\mathrm{opt}} \sim a_{0}(\rho)+\frac{a_{1}(\rho)}{M},
$$

where $a_{0}(\rho)$ and $a_{1}(\rho)$ are strictly positive functions of $\rho$ and $a_{0}(\rho)$ is the decreasing limit of $\xi_{\text {opt }}$ when $M \rightarrow+\infty$. A good approximation of $a_{0}(\rho)$, obtained by a least square approximation, is given by

$$
a_{0}(\rho)=\frac{\sqrt{\rho}}{\sum_{i=0}^{3} a_{0, i} \rho^{i}}, \frac{1}{128} \leq \rho \leq \frac{127}{128},
$$

with

$$
\begin{aligned}
& a_{0,0}=0.9067720699, \quad a_{0,1}=-0.001014926616 \\
& a_{0,2}=0.2787453767, \quad a_{0,3}=-0.08033355679
\end{aligned}
$$

In a similar way $\xi_{\text {dRRC }}$ is well approximated by

$$
\xi_{\mathrm{dRRC}} \sim b_{0}(\rho)+\frac{b_{1}(\rho)}{M},
$$

where $b_{0}(\rho)$ is strictly positive and is the decreasing limit of $\xi_{\mathrm{dRRC}}$. A good approximation of $b_{0}(\rho)$ is given by

$$
b_{0}(\rho)=\frac{\sqrt{\rho}}{\sum_{i=0}^{3} b_{0, i} \rho^{i}}, \frac{1}{128} \leq \rho \leq \frac{127}{128}
$$

with

$$
\begin{aligned}
& b_{0,0}=0.9073284361, \quad b_{0,1}=-0.006242158550 \\
& b_{0,2}=0.2853950713, \quad b_{0,3}=-0.05069930790
\end{aligned}
$$

Figure 4 gives the graphs of functions $a_{0}(\rho)$ and $b_{0}(\rho)$, usable only as approximations of the limits when $\frac{1}{128} \leq \rho \leq \frac{127}{128}$. It has to be noted that the OTFL curve is always above the $\mathrm{dRRC}$ one, though the difference is very small for low $\rho$ values. Note also that as long $\rho \geq \frac{1}{128}$, the limit is always above 0 . For instance with $\rho=\frac{9}{128}$, as for an LTE setting but assuming now $M$ tends to infinity, while we get $\xi_{\text {cp-ofdm }}=0$, the limits for the dRRC and OTFL are $\xi_{\mathrm{dRRC}}=b_{0}(9 / 128)=0.2919410401$ and $\xi_{\mathrm{opt}}=a_{0}(9 / 128)=0.2920156297$, respectively.

\section{Conclusion}

Various recent studies related to waveform design for $5 \mathrm{G}$ systems focus on very short time windows, i.e. with duration equal to one CP-OFDM symbol. These windows can be either implemented using time overlapping as, for WOLA-OFDM, or, as FMT systems, with no 


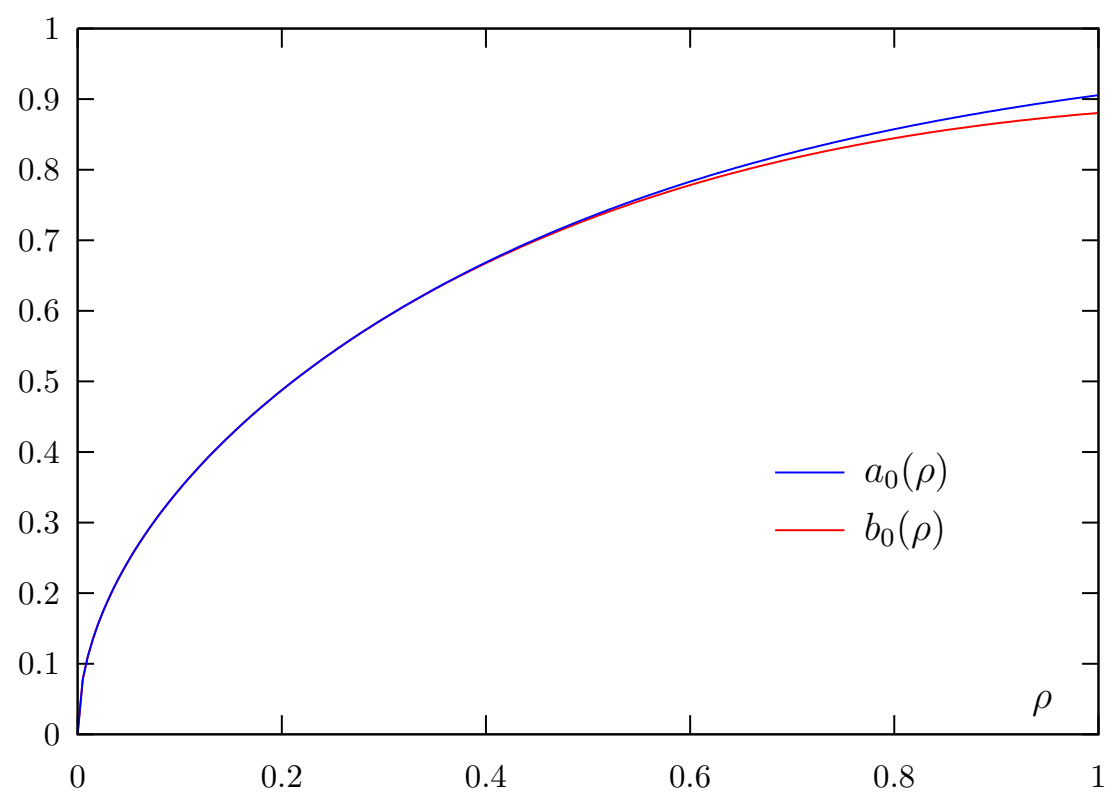

Figure 4: Limit functions $a_{0}(\rho)$ and $b_{0}(\rho)$ for $\xi_{\text {opt }}$ and $\xi_{\text {dRRC }}$, respectively.

overlapping in time. Differently from our previous publications $[6,7]$, the present work includes the case of FMT that do not necessarily reach a maximum spectral efficiency. Indeed, denoting by $M$ the subcarrier number and $N$ the decimation/expansion factor, the only restriction is given by $M+1 \leq N \leq 2 M-1$. In this paper, we have seen that the discretetime Root Raised Cosine function implemented in time, we have named dual RRC (dRRC), also provides perfect reconstruction FMT systems. Our aim being to provide optimal results for the time-frequency localization criterion, i.e. a key criterion for transmission through time-frequency dispersive transmission channels, we have analyzed the dRRC pulse in terms of TFL. To do so, we first adapted our compact representation (CR) method [11] to the case of a symmetrical function. It appeared that optimal windows, named OTFL, could be obtained with a low degree, at most $d=3$, of the symmetrical CR (SCR) meaning the number of optimization variables can be considerably reduced.

Our comparison between the OTFL and dRRC windows has shown that the dRRC has very good TFL features. First of all, we have proved that among all the short length PR FMT solutions, the dRRC prototype filter is optimal with respect to the minimization of the second order moment in frequency. Furthermore, when a high spectral efficiency is required, i.e. low values of the parameter $\rho=(N-M) / M$, as the case for LTE systems, the dRRC attains a nearly optimal TFL measure. Consequently, the interest of using an OTFL window only concerns the case of low spectral efficiency FMT systems and also, using a duality principle [10], the case of multicarrier faster than Nyquist (FTN) systems for high $\rho$ values, i.e. FTN systems nearly twice as fast as the conventional Nyquist rate. 


\section{References}

[1] METIS deliverable D1.1. Scenarios, requirements and KPIs for 5G mobile and wireless system, May 2013. Tech. Rep. ICT-317669.

[2] 5GNOW consortium D3.1. 5G waveform candidate selection. Nov. 2013.

[3] M. Renfors, X. Mestre, E. Kofidis, and F. Bader, editors. Orthogonal Waveforms and Filter Banks for Future Communication Systems. Elsevier, Academic Press, 2017.

[4] Qualcomm Technologies. 5G Waveform and multiple access techniques, 2015.

[5] R. Zayani, Y. Medjahdi, H. Shaiek, and D. Roviras. WOLA-OFDM: a potential candidate for asynchronous 5G. In GLOBECOM 2016, pages -, Washington, USA, December 2016.

[6] D. Pinchon and P. Siohan. Closed form expression of optimal short PR FMT prototype filters. In Proceedings Globecom'11 (Houston, USA), December 2011.

[7] D. Pinchon and P. Siohan. Derivation of analytical expressions for flexible PR low complexity FBMC systems. In Proc. EUSIPCO 2013, Marrakech, Morocco, sep 2013.

[8] D. Roque and C. Siclet. Performances of Weighted Cyclic Prefix OFDM with low complexity equalization. IEEE Communications Letters, 17(3):439-442, 2013.

[9] Z. Zhao, M. Schellmann, X. Gong, Q. Wang, R. Böhnke, and Y. Guo. Pulse shaping design for OFDM systems. EURASIP Journal on Wireless Communications and Networking, 2017(1):74, Apr 2017.

[10] A. Marquet, D. Roque, C. Siclet, and P. Siohan. FTN multicarrier transmission based on tight Gabor frames. EURASIP Journal on Wireless Communications and Networking, 2017(1):97, May 2017.

[11] D. Pinchon, P. Siohan, and C. Siclet. Design techniques for orthogonal modulated filter banks based on a compact representation. IEEE Transactions on Signal Processing, 52(6):1682-1692, June 2004.

[12] G. Cherubini, E. Eleftheriou, and S. Olcer. Filtered multitone modulation for VDSL. In Proc. of IEEE Global Telecommunications Conference, pages 1139-1144, 1999.

[13] M.I. Doroslovački. Product of second moments in time and frequency for discrete-time signals and the uncertainty limit. Signal Processing, 67(1):59-76, 1998.

[14] Ivan S. Gaspar, Luciano L. Mendes, Nicola Michailow, and Gerhard Fettweis. A synchronization technique for generalized frequency division multiplexing. EURASIP Journal on Advances in Signal Processing, 2014(1):67, May 2014. 
[15] C. Lawrence, J.L. Zhou, and A.L. Tits. User's guide for CFSQP version 2.5: A C code for solving (large scale) constrained nonlinear (minimax) optimization problems, generating iterates satisfying all inequality constraints. Technical Report TR-94-16r1, University of Maryland, 1998.

[16] P. Siohan and C. Roche. Cosine modulated filterbanks based on Extended Gaussian Functions. IEEE Transactions on Signal Processing, 48(11):3052-3061, November 2000. 


\section{A Proof of theorem 4.2}

Let $P(z)$ be in $\mathcal{P}_{M, N}$. As explained before, perfect reconstruction relations

$$
p[n]^{2}+p[n+M]^{2}=1,0 \leq n \leq N-M-1, \quad p[n]^{2}=1, N-M \leq n \leq M-1,
$$

impose that there exist $N-M$ angles $\theta_{n}, \quad 0 \leq n \leq N-M-1$ and $2 M-N$ numbers $\varepsilon_{n}, N-M \leq n \leq M-1$ equal to \pm 1 such that

$$
\begin{aligned}
p[n] & =\sin \theta_{n}, \quad 0 \leq n \leq N-M-1, \\
p[n+M] & =\cos \theta_{n}, \quad 0 \leq n \leq N-M-1, \\
p[n] & =\varepsilon_{n}, \quad N-M \leq n \leq M-1 .
\end{aligned}
$$

The numerator $A$ of $M_{2}$ is given by $A=\sum_{n}(p[n]-p[n-1])^{2}$.

In a first reduction step, we prove that the minimum of $A$ may be obtained by choosing $0 \leq \theta_{n} \leq \frac{\pi}{2}, 0 \leq n \leq N-M-1$ and $\varepsilon_{n}=1, N-M \leq n \leq M-1$.

By periodicity, we may suppose that $\theta_{n}$ angles verify $-\pi<\theta_{n} \leq \pi$ for $0 \leq n \leq N-M-1$.

In the following, for any angle $\theta$, we denote by $\bar{\theta}$ the angle such that $-\pi<\bar{\theta} \leq \pi$ and $\theta-\bar{\theta}$ is a multiple of $2 \pi$.

If $p[0]=\sin \theta_{0}<0$, i.e. $-\pi<\theta<0$, then we may replace all angles in such a way that their sine and cosine have an opposite sign, i. e. $\theta_{n} \rightarrow \overline{\theta_{n}+\pi}, 0 \leq n \leq N-M-1$, and the sign of $\varepsilon_{n}, N-M \leq n \leq M-1$ is also changed. It is like changing the sign of $P(z)$ and this does not modify the value of $A$. We may thus suppose that $0 \leq \theta_{0} \leq \pi$.

Let us now suppose that there exists $k$ with $0<k \leq N-1$ such that $p[n]>0$ for $0 \leq n<k$ and $p[k]<0$. By changing the sign of $p[n], k \leq n \leq N-1$, and keeping the sign of $p \mid n], 0 \leq n<k$, we only modify one term, $(p[k-1]-p[k])^{2}$ in $A$, which is decreased. Three situations are then possible.

- For $0<k \leq N-M-1, \theta_{n} \rightarrow \overline{\pi-\theta_{n}}, 0 \leq n<k, \theta_{n} \rightarrow \overline{\pi+\theta_{n}}, k \leq n<N-M-1$ and $\varepsilon_{n} \rightarrow-\varepsilon_{n}, N-M \leq n \leq M-1$,

- For $N-M \leq k \leq M-1, \theta_{n} \rightarrow \overline{\pi-\theta_{n}}, 0 \leq n<N-1$ and $\varepsilon_{n} \rightarrow-\varepsilon_{n}, k \leq n \leq M-1$,

- For $M \leq k \leq N-1, \theta_{n} \rightarrow \overline{\pi-\theta_{n}}, k-M \leq n \leq N-M-1$

At the end of this process, we get $p[n] \geq 0$, and in particular $\sin \theta_{n} \geq 0, \cos \theta_{n}>0$, that is $0 \leq \theta_{n} \leq \frac{\pi}{2}, 0 \leq n \leq N-M-1$, and $\epsilon_{n}=1, N-M \leq n \leq M$.

With these conditions $A$ is equal to

$$
\begin{aligned}
A= & \sin ^{2} \theta_{0}+\sum_{n=1}^{N-M-1}\left(\sin \theta_{n}-\sin \theta_{n-1}\right)^{2}+\left(1-\sin \theta_{N-M-1}\right)^{2} \\
& +\left(\cos \theta_{0}-1\right)^{2}+\sum_{n=1}^{N-M-1}\left(\cos \theta_{n}-\cos \theta_{n-1}\right)^{2}+\cos ^{2} \theta_{N-M-1},
\end{aligned}
$$

which is equivalent to

$$
A=2(N-M+1)-2 \cos \theta_{0}-2 \sum_{n=1}^{N-M-1} \cos \left(\theta_{n}-\theta_{n-1}\right)-2 \sin \theta_{N-M-1} .
$$


As a function of $\theta_{n}, 0 \leq n \leq N-M-1, A$ is a positive regular function that reaches its minima on points where its partial derivatives vanish. Some global minima are located in the set $\left[0, \frac{\pi}{2}\right]^{N-M}$ thanks to the reduction argument of the first step of the proof, and they necessarily correspond to a null gradient for $A$.

Straightforward computations give

$$
\begin{aligned}
\frac{1}{2} \frac{\partial A}{\partial \theta_{0}} & =\sin \theta_{0}+\sin \left(\theta_{0}-\theta_{1}\right)=2 \sin \left(\theta_{0}-\frac{\theta_{1}}{2}\right) \cos \frac{\theta_{1}}{2}, \\
\frac{1}{2} \frac{\partial A}{\partial \theta_{n}} & =\sin \left(\theta_{n}-\theta_{n+1}\right)-\sin \left(\theta_{n-1}-\theta_{n}\right) \\
& =2 \sin \left(\theta_{n}-\frac{\theta_{n-1}+\theta_{n+1}}{2}\right) \cos \left(\frac{\theta_{n+1}-\theta_{n-1}}{2}\right), 1 \leq n \leq N-M-2, \\
\frac{1}{2} \frac{\partial A}{\partial \theta_{N-M-1}} & =-\cos \theta_{N-M-1}-\sin \left(\theta_{N-M-2}-\theta_{N-M-1}\right) \\
& =-2 \sin \left(\frac{\pi}{4}-\theta_{N-M-1}+\frac{\theta_{N-M-2}}{2}\right) \cos \left(\frac{\pi}{4}-\frac{\theta_{N-M-2}}{2}\right) .
\end{aligned}
$$

In equation (27), $0 \leq \theta_{1} \leq \frac{\pi}{4}$ and thus $\cos \frac{\theta_{1}}{2} \neq 0$. To cancel $\frac{1}{2} \frac{\partial A}{\partial \theta_{0}}$ we must have $\sin \left(\theta_{0}-\frac{\theta_{1}}{2}\right)=0$ and because $-\frac{\pi}{4} \leq \theta_{0}-\frac{\theta_{1}}{2} \leq \frac{3 \pi}{4}$, we get $\theta_{0}-\frac{\theta_{1}}{2}=0$, otherwise said $\theta_{1}=2 \theta_{0}$.

Let us now prove by recurrence that $\theta_{n}=(n+1) \theta_{0}$ for $1 \leq n \leq N-M-1$. This is true for $n=1$. Let us suppose that it is also true for $1 \leq k \leq n$ with $1 \leq n<N-M-1$. Cancellation of $\frac{1}{2} \frac{\partial A}{\partial \theta_{n}}$ given relation (28) then requires either $\cos \left(\frac{\theta_{n+1}-\theta_{n-1}}{2}\right)=0$, but because $-\frac{\pi}{4} \leq \frac{\theta_{n+1}-\theta_{n-1}}{2} \leq \frac{\pi}{4}$, this is impossible, or $\sin \left(\theta_{n}-\frac{\theta_{n-1}+\theta_{n+1}}{2}\right)=0$. As $-\frac{\pi}{4} \leq \theta_{n}-\frac{\theta_{n-1}+\theta_{n+1}}{2} \leq$ $\frac{3 \pi}{4}$, we get $\theta_{n}-\frac{\theta_{n-1}+\theta_{n+1}}{2}=0$. The recurrence hypothesis then gives $\theta_{n-1}=n \theta_{0}$ and $\theta_{n}=(n+1) \theta_{0}$ and thus $\theta_{n+1}=2 \theta_{n}-\theta_{n-1}=(n+2) \theta_{0}$.

As $-\frac{\pi}{4} \leq \frac{\pi}{4}-\theta_{N-M-1}+\frac{\theta_{N-M-2}}{2} \leq \frac{3 \pi}{4}$ and $-\frac{\pi}{4} \leq \frac{\pi}{4}-\frac{\theta_{N-M-2}}{2} \leq \frac{3 \pi}{4}, \frac{1}{2} \frac{\partial A}{\partial \theta_{N-M-1}}$, given by relation (29), only cancels if $\frac{\pi}{4}-\theta_{N-M-1}+\frac{\theta_{N-M-2}}{2}=0$. Because $\theta_{N-M-1}=(N-M) \theta_{0}$ and $\theta_{N-M-2}=(N-M-1) \theta_{0}$, we get $\theta_{0}=\frac{\pi}{2(N-M+1)}$ and thus

$$
\theta_{n}=\frac{(n+1) \pi}{2(N-M+1)}, 0 \leq n \leq N-M-1 .
$$

The only filter $P(z)$ that realizes the minimum of $M_{2}$ is therefore the dRRC filter.

Reporting those values of $\theta_{n}$ in expression (26), we get

$$
\begin{aligned}
A & =2(N-M+1)-2(N-M) \cos \frac{\pi}{2(N-M+1)}-2 \sin \frac{(N-M) \pi}{2(N-M+1)}, \\
& =2(N-M+1)\left(1-\cos \frac{\pi}{2(N-M+1)}\right) \\
& =4(N-M+1) \sin ^{2} \frac{\pi}{4(N-M+1)} .
\end{aligned}
$$

In expression (12) of $M_{2}$ the denominator is equal to $M$ and thus equality (18) is proven. 